\title{
Malakoplakia of the prostate masquerading as locally advanced prostate cancer on mpMRI
}

\author{
Robert Thomas Dale, $M D^{\prime}$; Michael Metcalfe, $M D^{\prime}$; Silvia Chang, $M D^{2}$; Edward Jones, $M D^{3}$; Peter Black, $M D^{\prime}$
}

'Department of Urologic Sciences, University of British Columbia (UBC); ${ }^{2}$ Department of Radiology, UBC; 32Department of Pathology and Laboratory Science, UBC; Vancouver, BC, Canada

Cite as: Can Urol Assoc J 2015;9(11-12):E910-2. http://dx.doi.org/10.5489/cuai.3235 Published online December 14, 2015.

\section{Abstract}

A 66-year-old man was referred for urological evaluation for an abnormal digital rectal exam (cT2a, subtle nodule at left base, $121 \mathrm{cc}$ prostate) and an elevated prostate specific antigen (PSA) of $8.0 \mathrm{ng} / \mathrm{ml}$. Subsequent 12-core transrectal ultrasound (TRUS)guided biopsy revealed Gleason $3+4$ adenocarcinoma in seven of 12 cores, including all six cores on the right side and one core at the left apex. No extraprostatic extension was identified. Postbiopsy, the patient developed urinary retention requiring a catheter, as well as an Escherichia coli (E. coli) urinary tract infection (UTI) requiring hospitalization and intravenous antibiotics.

\section{Case report}

A 66-year-old man was referred for urological evaluation for an abnormal digital rectal exam (cT2a, subtle nodule at left base, 121 cc prostate) and an elevated PSA of $8.0 \mathrm{ng} / \mathrm{ml}$. Subsequent 12-core TRUS-guided biopsy revealed Gleason $3+4$ adenocarcinoma in seven of 12 cores, including all six cores on the right side and one core at the left apex. No extraprostatic extension was identified. Post-biopsy, the patient developed urinary retention requiring a catheter, as well as an $E$. coli UTI requiring hospitalization and intravenous antibiotics.

A staging multiparametric MRI (mpMRI) was performed six weeks after biopsy in an attempt to address the discordance between clinical exam and biopsy findings with respect to lesion location. MpMRI was obtained using a 3-Tesla scanner and a multi-phased array body surface coil without an endorectal coil. Image sequences acquired included tri-planar high-resolution T2-weighted imaging, diffusion-weighted imaging (DWI) and high-temporal resolution dynamic contrast enhanced (DCE) imaging. DWI was performed at b-values of 50,500, and 1000, with an apparent diffusion coefficient (ADC) map generated. DCE was performed after intravenous administration of $0.1 \mathrm{mmol} / \mathrm{kg}$ of gadobutrol by a power injector with 60 data acquisitions at a temporal resolution of $<10 \mathrm{~s}$. This mpMRI demonstrated a $4.5 \times 2 \mathrm{~cm}$ enhancing soft tissue mass with strong restricted diffusion and loss of fat plane with rectum arising from the left mid-prostate. A second foci, measuring $2.1 \times 1.5 \mathrm{~cm}$ with ADC maps and showing diffuse strongly restricted diffusion, was seen in the right base with invasion into the seminal vesicle (Fig. 1). All areas demonstrated enhancement with minimal washout on DCE.

After discussion with the patient regarding the discordant exam, biopsy and MRI findings with respect to location and extent of tumour, and after consultation by both urological and radiation oncologists to consider all treatment options, the patient chose radical prostatectomy.

Bilateral, wide-field, robotic-assisted laparoscopic radical prostatectomy with bilateral pelvic lymph node dissection was performed. Intraoperatively, there was significant fibrosis of the posterior rectal plane on the left side extending down into the pararectal gutter, but the prostate was separable from the rectum with sharp dissection. No complications were encountered.

Pathology revealed pT2cNOMO Gleason $3+3=6$ adenocarcinoma involving $20 \%$ of the prostate, primarily in the right peripheral zone from apex to base. No extraprostatic extension or seminal vesicle invasion was identified and the surgical margins were negative. Instead, mixed-pattern prostatitis with malakoplakia extending into extraprostatic tissue bilaterally was observed. The right seminal vesicle demonstrated acute on chronic prostatitis with microabcesses (Fig. 2). Prussian blue, von Kossa, and PAS/D stains were used to visualize Michaelis-Gutmann bodies. ${ }^{1}$ 

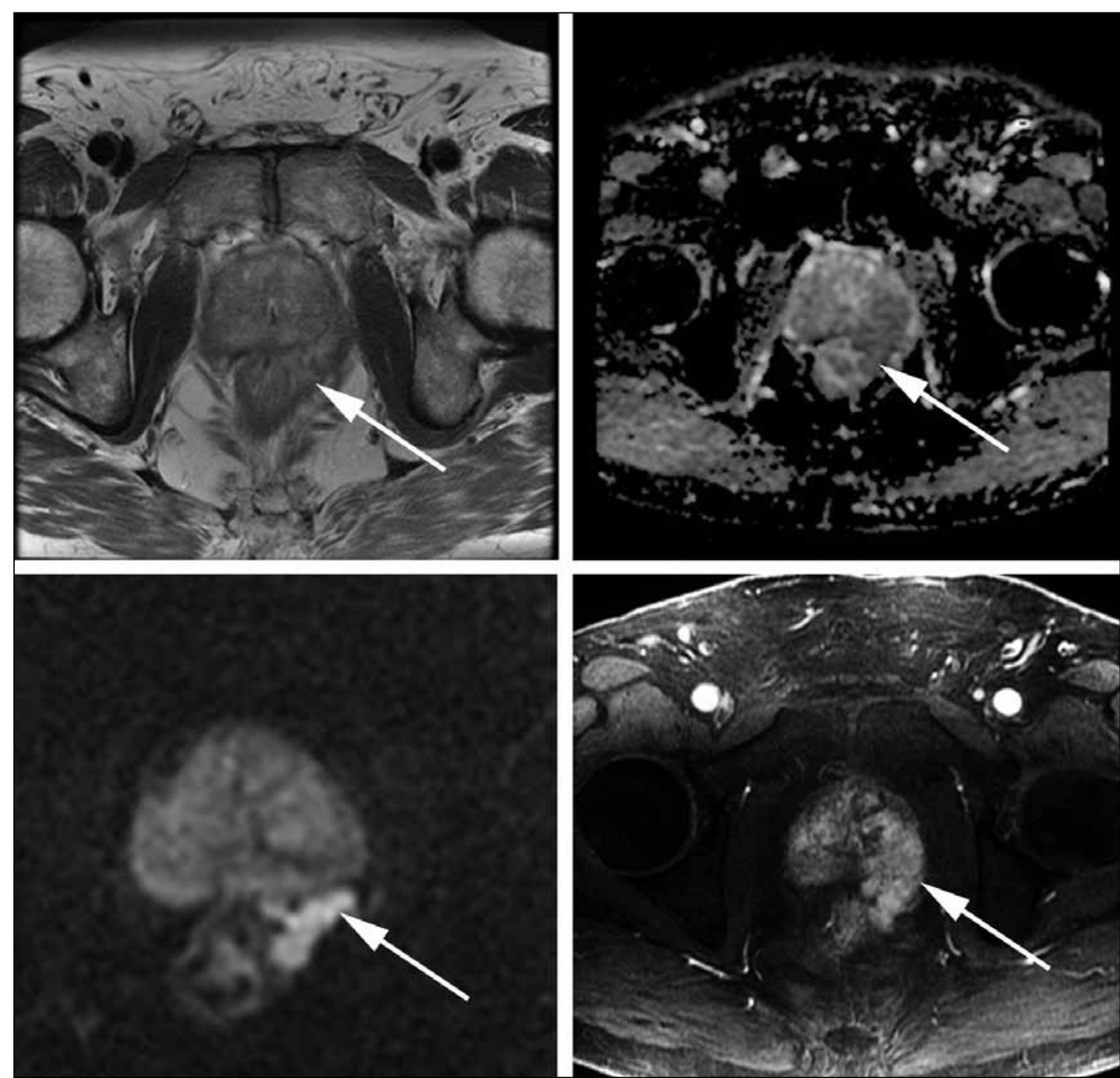

Fig. 1. Axial T2 (upper left), $A D C$ map (upper right) and DWI (lower left) slices from $3 T$-multiparametric MRI demonstrating a mass with restricted diffusion (arrows) from the left mid-gland of the prostate extending beyond the capsule to the rectum, with loss of the intervening fat plane. An image from the dynamic contrast enhancement series (bottom right) demonstrates hypervascular enhancement of the lesion (arrow).

\section{Discussion}

This case highlights a rare differential diagnosis to be considered in the context of abnormal findings on mpMRI of the prostate. To our knowledge, this is the first report describing prostatic malakoplakia on mpMRI. Even in retrospect the findings were ndistinguishable from locally advanced prostate cancer with low intensity, irregular signal in the peripheral zone on $\mathrm{T} 2$, severe restriction on DWI, and rapid contrast uptake and washout on DCE.

MpMRI is evolving as a standard tool for the diagnosis and staging of prostate cancer. It is considered to have adequate positive predictive value in the detection of extraprostatic extension of the prostate cancer, but insufficient negative predictive value to be adopted uniformly for presurgical planning. (0.84 and 0.68 , respectively in intermediate risk patients). ${ }^{2}$

The mpMRI findings in the current patient by themselves were consistent with locally advanced disease, but these were clearly discrepant from the biopsy findings, which showed minimal cancer on the left side of the prostate, where the tumour was described as abutting the rectum. The patient was keen to avoid radiation, and, with chronic obstructive voiding symptoms due to a very large prostate, was most interested in surgical resection. With some trepidation due to the imaging findings, the robotic prostatectomy was performed and allowed us to determine the true diagnosis. We recognize the rarity of the case, with less than 1000 patients diagnosed with malakoplakia per year in the U.S. ${ }^{1,3-}$ ${ }^{5}$ The patient's history of significant post-UTI with urinary 


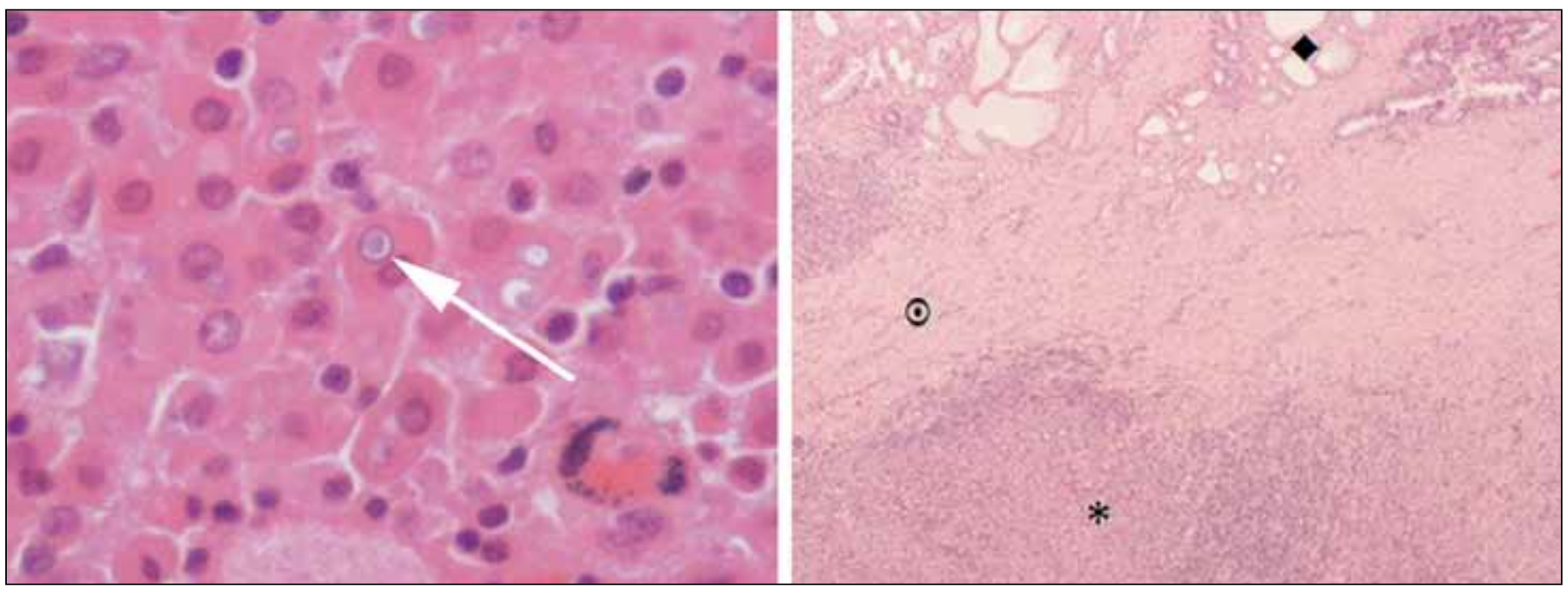

Fig. 2. Typical histological features of malakoplakia, including Michaelis-Gutmann bodies within prostatic histiocytes (left). The low-power view (right) demonstrates malakoplakia inflammation $(*)$, normal prostatic capsule $(\odot)$, and adenocarcinoma $(\diamond)$.

retention was a key clinical clue to the possible etiology, although the rarity of such a diagnosis would preclude any change in approach to such a patient. However, with rising rates of infectious complications after prostate biopsy, the incidence may rise. ${ }^{6,7}$ We highlight the potential for this or other rare inflammatory or infectious conditions to occur and to lead falsely to a diagnosis of locally advanced prostate cancer, with impact on subsequent choice of therapy.

Competing interests: The authors declare no competing financial or personal interests.

This paper has been peer-reviewed.

\section{References}

1. Wagner D, Joseph J, Huang J, et al. Malakoplakia of the prostate on needle core biopsy: A case report and review of the literature. Int I Surg Pathol 2007;15:86-9. http://dx.doi. org/10.1177/1066896906295892

2. Hoeks CMA, Barentsz J0, Hambrock T, et al. Prostate cancer: Multiparametric MR imaging for detection, localization, and staging. Radiology 2011;261:46-66. http://dx.doi.org/10.1148/radiol.11091822

3. Michaelis L, Gutmann C.Uber einschlusse in blasentumoren. Ztschr Klin Med 1902;47:208-15.

4. Dasgupta P, Womack C, Turner A, et al. Malacoplakia: von Hansemann's disease. BJU Int 2001;84: 464-9. http://dx.doi.org/10.1046/i.1464-410x.1999.00198.x

5. Stanton M, Maxted W. Malacoplakia: A study of the literature and current concepts of pathogenesis, diagnosis and treatment. J Urol 1982;125:139-46.

6. Rudzinski JK, Kawakami J. Incidence of infectious complications following transrectal ultrasound-guided prostate biopsy in Calgary, Alberta, Canada: A retrospective population-based analysis. Can Urol Assoc J 2014;8:E301-5. http://dx.doi.org/10.5489/cuaj.1751

7. Loeb S, Carter HB, Berndt SI, et al. Complications after prostate biopsy: Data from SEERMedicare. J Urol 2011;186:1830-4. http://dx.doi.org/10.1016/i.juro.2011.06.057

Correspondence: Dr. Robert Thomas Dale, Department of Urologic Sciences, University of British Columbia, Vancouver, BC, Canada; robert.t.dale@gmail.com 